\title{
Economic receding horizon control without terminal constraints *
}

\author{
Lars Grüne $^{\text {a }}$ \\ ${ }^{a}$ Mathematisches Institut, Universität Bayreuth, 95440 Bayreuth, Germany
}

\begin{abstract}
We consider a receding horizon control scheme without terminal constraints in which the stage cost is defined by economic criteria, i.e., not necessarily linked to a stabilization or tracking problem. We analyze the performance of the resulting receding horizon controller with a particular focus on the case of optimal steady states for the corresponding averaged infinite horizon problem. Using a turnpike property and suitable controllability properties we prove near optimal performance of the controller and convergence of the closed loop solution to a neighborhood of the optimal steady state. Two examples illustrate our findings numerically and show how to verify the imposed assumptions.
\end{abstract}

Key words: economic MPC, turnpike property, controllability.

\section{Introduction}

In this paper we investigate the performance of receding horizon control schemes with general stage costs. In receding horizon control — often also called model predictive control (MPC) - a feedback law is synthesized from the first elements of finite horizon optimal control sequences which are iteratively computed along the closed loop solution. This procedure has by now become a standard method for the optimization based stabilization and tracking control. In stabilization problems, the stage cost typically penalizes the distance to a desired equilibrium or time varying reference solution. While there is an ample literature on the analysis of stabilizing receding horizon schemes - see, e.g., the survey paper [9] or the monographs $[8,12]$ and the extensive lists of references therein - results for stage costs not related to stabilization and tracking are much more scarce. Due to the fact that such a stage cost usually reflects an economic criterion rather than a distance to a reference, they are often called economic MPC or economic receding horizon control.

The receding horizon approach to economic problems

\footnotetext{
* This paper was not presented at any IFAC meeting. Corresponding author L. Grüne. Tel. +49-921-553270. Fax $+49-921-555361$. Supported by the European Union under the 7th Framework Programme FP7-PEOPLE-2010-ITN, Grant agreement number 264735-SADCO
}

Email address: lars.gruene@uni-bayreuth.de (Lars Grüne). is on the one hand appealing because it naturally yields a control function in feedback form. Another advantage is its ability to solve infinite horizon optimal control problems numerically with much lower computational effort than classical approaches like, e.g., dynamic programming. Indeed, while the online computatonal effort of receding horizon schemes is higher, it typically scales much more moderately with the state dimension than the offline computational effort of dynamic programming and is thus less affected by the curse of dimensionality. As examples, e.g., in [8] show, using state-of-the-art optimization algorithms, MPC is nowadays able to handle even discretized PDE models with hundreds of state variables with satisfactory accuracy in reasonable time.

When using receding horizon control in order to reduce the computational burden attached to infinite horizon optimal control problems, the question whether the resulting solution approximates the infinite horizon optimal solution becomes important. Indeed, while research in stabilizing MPC is often focused on issues like stability and feasibility, approximate optimality is the natural property to look at when the main objective is the optimization of a given cost criterion. While stability-like properties like the convergence to optimal steady states are of interest in economic problems, too, they are in general not a meaningful criterion on their own but only an additional feature once near optimal performance can be ensured. For economic receding horizon control, these issues have recently been investigated in $[1-3,6]$. The central idea of the schemes in these references is as follows: first, one determines an optimal equilibrium or periodic 
orbit for the infinite horizon averaged problem and then this solution is used as a terminal constraint for the finite horizon optimal control problem to be solved in each step of the receding horizon scheme.

In contrast to these references, in this paper we do not impose any terminal constraints. Thus, we investigate whether a receding horizon control scheme is able to find an optimal operating point or orbit without providing it as additional information to the algorithm. The motivation for this study is that without terminal constraints the amount of preparatory computations is reduced (since the knowledge of the optimal solution is only needed for the analysis but not for running the scheme), that the absence of terminal constraints may make the optimal control problem in each step easier to solve and that it may lead to a larger operating region of the resulting controller, because the feasible region will typically increase.

The price we pay for removing the terminal constraints is on the one hand a more involved analysis using stronger assumptions on the underlying finite horizon problems. To this end, we provide sufficient conditions based on certain controllability assumptions and on the turnpike property, which is a classical tool in optimal control [5, Section 4.4], particularly for understanding the optimal dynamics of economic control problems [10]. On the other hand, our approach only yields approximate optimal performance instead of exact optimal performance as in [1-3]. However, we will prove that the performance converges to the optimal one as the receding optimization horizon grows and by numerical simulations we illustrate that this convergence may even be exponentially fast. Moreover, the conditions we impose allow to prove approximate optimality of the receding horizon closed loop not only on the infinite horizon but also during the transient phase, i.e., on the finite time interval until a neighborhood of the optimal steady state is reached. To the best of our knowledge results on approximately optimal transient behavior have not been obtained before in the economic MPC literature. While our general results are formulated in an abstract setting, for the derivation of checkable sufficient conditions we focus on the particular case of optimal equilibria. These conditions hold for general nonlinear systems, but become particularly convenient for linear dynamics, cf. Remark 6.5.

The paper is organized as follows. After formulating the problem and premilinary results in Section 2 we discuss two motivating examples in Section 3 which help to identify reasonable conditions to be imposed in the subsequent sections. General results on value convergence are given in Section 4. The conditions imposed in Section 4 are further investigated in Sections 5 and 6 for the case of optimal steady states. Here we derive checkable sufficient conditions based on a turnpike property and suitable controllability conditions. In Section 7 we investigate the limiting behavior of the receding horizon closed loop and optimality during the transient phase. Finally, Section 8 concludes the paper.

\section{Problem formulation and preliminaries}

We consider discrete time control systems with state $x \in X$ and control values $u \in U$, where $X$ and $U$ are normed spaces with norms denoted by $\|\cdot\|$. The control system under consideration is given by

$$
x(k+1)=f(x(k), u(k))
$$

with $f: X \times U \rightarrow X$. For a given control sequence $u=(u(0), \ldots, u(K-1)) \in U^{K}$ or $u=(u(0), u(1), \ldots) \in$ $U^{\infty}$, by $x_{u}(k, x)$ we denote the solution of (1) with initial value $x=x_{u}(0, x) \in X$.

For given admissible sets of states $\mathbb{X} \subseteq X$ and control values $\mathbb{U} \subseteq U$ and an initial value $x \in \mathbb{X}$ we call the control sequences $u \in \mathbb{U}^{K}$ satisfying $x_{u}(k, x) \in \mathbb{X}$ for all $k=0, \ldots, K$ admissible. The set of all admissible control sequences is denoted by $\mathbb{U}^{K}(x)$. Similarly, we define the set $\mathbb{U}^{\infty}(x)$ of admissible control sequences of infinite length. For simplicity of exposition we assume $\mathbb{U}^{\infty}(x) \neq \emptyset$ for all $x \in \mathbb{X}$, i.e., that for each initial value $x \in \mathbb{X}$ we can find a trajectory staying inside $\mathbb{X}$ for all future times. This condition may be relaxed if desired, using, e.g., results from [8, Sections 8.2-8.3] or [11].

Given a feedback map $\mu: X \rightarrow \mathbb{U}$, we denote the solutions of the closed loop system $x(k+1)=$ $f(x(k), \mu(x(k)))$ by $x_{\mu}(k)$ or by $x_{\mu}(k, x)$ if we want to emphasize the dependence on the initial value $x=x_{\mu}(0)$. We say that a feedback law $\mu$ is admissible if $f(x, \mu(x)) \in \mathbb{X}$ holds for all $x \in \mathbb{X}$.

Our goal is now to find an admissible feedback controller which yields trajectories with guaranteed bounds on the average cost, preferably as small as possible. To this end, for a given running cost $\ell: X \times U \rightarrow \mathbb{R}$ we define the averaged functionals

$$
\begin{aligned}
J_{N}(x, u) & :=\frac{1}{N} \sum_{k=0}^{N-1} \ell\left(x_{u}(k, x), u(k)\right), \\
J_{\infty}(x, u) & :=\limsup _{N \rightarrow \infty} J_{N}(x, u)
\end{aligned}
$$

as well as the optimal value functions $V_{N}(x):=$ $\inf _{u \in \mathbb{U}^{N}(x)} J_{N}(x, u)$ and $V_{\infty}(x):=\inf _{u \in \mathbb{U}^{\infty}(x)} J_{\infty}(x, u)$. Here we assume that $\ell$ is bounded from below on $\mathbb{X}$, i.e., that $\ell_{\min }:=\inf _{x \in \mathbb{X}, u \in \mathbb{U}} \ell(x, u)$ is finite. This assumption immediately yields $J_{N}(x, u) \geq \ell_{\min }$ and $J_{\infty}(x, u) \geq \ell_{\min }$ for all admissible control sequences. In order to simplify the exposition in what follows, we assume that for each $x \in \mathbb{X}$ a (not necessarily unique) optimal control sequence $u_{N, x}^{*} \in \mathbb{U}^{N}(x)$ for $J_{N}$ exist, i.e., a sequence satisfying $V_{N}(x)=J_{N}\left(x, u_{N, x}^{*}\right)$.

Similarly to the open loop functionals, we can define the average cost of the closed loop solution for any feedback law $\mu$ by

$$
J_{K}^{c l}(x, \mu):=\frac{1}{K} \sum_{k=0}^{K-1} \ell\left(x_{\mu}(k, x), \mu\left(x_{\mu}(k, x)\right)\right)
$$


and $J_{\infty}^{c l}(x, \mu):=\lim \sup _{K \rightarrow \infty} J_{K}(x, \mu)$. In order to find a feedback $\mu$ we will apply a receding horizon control scheme, also known as model predictive control (MPC). This method consists of solving the open loop optimization problem of minimizing $J_{N}(x, u)$ with initial value $x=x_{\mu}(k)$ at each sampling instant $k$ for some given optimization horizon $N \in \mathbb{N}$ and then defining the feedback value $\mu(x)=\mu_{N}(x)$ to be the first element of the corresponding optimal control sequence, i.e.,

$$
\mu_{N}(x)=u_{N, x}^{*}(0)
$$

Since nowadays efficient algorithms for the necessary online minimization of $J_{N}(x, u)$ are available (see, e.g., [8, Chapter 10]), this method is computationally feasible for large classes of systems.

Our goal in this paper is to derive upper bounds for $J_{K}^{c l}\left(x, \mu_{N}\right)$ and $J_{\infty}^{c l}\left(x, \mu_{N}\right)$ depending on the optimization horizon $N$. While the upper bounds we deduce are in general not necessarily optimal, we are able to identify certain situations in which they actually are. Particularly, in the presence of optimal equilibria we can formulate checkable sufficient conditions for this property which are linked to the classical turnpike property and certain controllability assumptions, cf. Sections 5 and 6 .

We end this section by introducing some basic notation and preliminary results. For subsets $\mathbb{Y} \subset X$ we denote the distance of a point $x \in X$ to $\mathbb{Y}$ by $|x|_{\mathbb{Y}}:=$ $\inf _{y \in \mathbb{Y}}\|x-y\|$. The open ball with radius $\delta>0$ around a set $\mathbb{Y} \subset X$ will be denoted by $\mathcal{B}_{\delta}(\mathbb{Y}):=\left\{\left.x \in X|| x\right|_{\mathbb{Y}}<\right.$ $\delta\}$ and for $\mathbb{Y}=\{y\}$ we write $\mathcal{B}_{\delta}(y)$ instead of $\mathcal{B}_{\delta}(\mathbb{Y})$. With $\mathcal{K}_{\infty}$ we denote the set of continuous functions $\alpha: \mathbb{R}_{0}^{+} \rightarrow \mathbb{R}_{0}^{+}$which are strictly increasing and unbounded with $\alpha(0)=0$. With $\mathcal{L}_{\mathbb{N}}$ we denote the set of functions $\delta: \mathbb{N} \rightarrow \mathbb{R}_{0}^{+}$which are (not necessarily strictly) decreasing with $\lim _{k \rightarrow \infty} \delta(k)=0$.

In our analysis we will make extensive use of the dynamic programming principle, cf. [4]. The form of this principle which applies here states that for the optimal control sequence $u_{N, x}^{*}$ for the problem with finite horizon $N$ and each $K \in\{1, \ldots, N-1\}$ the equality

$$
\begin{aligned}
V_{N}(x)= & \frac{1}{N} \sum_{k=0}^{K-1} \ell\left(x_{u_{N, x}^{*}}(k, x), u_{N, x}^{*}(k)\right) \\
& +\frac{N-K}{N} V_{N-K}\left(x_{u_{N, x}^{*}}(K, x)\right)
\end{aligned}
$$

holds. As a consequence, for $\mu_{N}(x)=u_{N, x}^{*}(0)$ we get $V_{N}(x)=\ell\left(x, \mu_{N}(x)\right) / N+(N-1) V_{N-1}\left(f\left(x, \mu_{N}(x)\right)\right) / N$. This implies the equation

$$
\ell\left(x, \mu_{N}(x)\right)=N V_{N}(x)-(N-1) V_{N-1}\left(f\left(x, \mu_{N}(x)\right)\right) .
$$

\section{Motivating examples}

In order to illustrate how receding horizon control without terminal constraints performs for problems with economic cost, we look at two motivating examples. All simulations were carried out with the MATLAB routine nmpc.m (cf. [8, Appendix A] and www.nmpc-book.com) which uses the fmincon optimization routine.

Example 3.1 (see also [7]) Consider the control system $x(k+1)=2 x(k)+u(k)$ with $X=U=\mathbb{R}$ and $\mathbb{U}=[-2,2]$. The running cost $\ell$ is chosen such that the control effort is penalized quadratically, i.e., $\ell(x, u)=u^{2}$ and we consider the admissible sets $\mathbb{X}=[-a, a]$ with $a=0.5$ and $a=1$. Hence, the optimal control problem tries to keep the system inside $\mathbb{X}$ with minimal average control effort. It is easily seen that an optimal way of doing this is to steer the system to the equilibrium $x^{e}=0$ in a finite number of steps $k^{\prime}$ and set $u(k)=u^{e}=0$ for $k \geq k^{\prime}$ which leads to $J_{\infty}(x, u)=0$. Since $\ell(x, u) \geq 0$ for all $x$ and $u$, this is the optimal value of $J_{\infty}$, i.e., $V_{\infty}(x)=0$ for all $x \in \mathbb{X}$.

Figure 1 shows the MPC closed loop trajectory $x(k)=$ $x_{\mu_{N}}(k, x)$ for $x=0.5$ (solid) and the open loop optimal trajectories $x_{u_{N, x(k)}}(\cdot, x(k))$ for each $k$ (dashed) for $\mathbb{X}=[-0.5,0.5]$. One sees that while the open loop trajectories eventually move to the upper boundary of the admissible set, the closed loop trajectory tends towards a neighborhood of $x^{e}=0$.

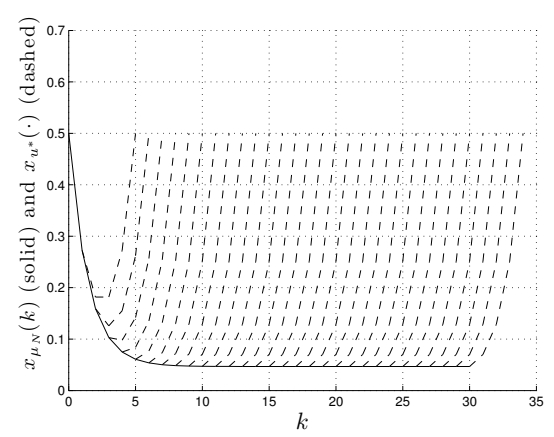

Fig. 1. Closed loop trajectory $x(k)=x_{\mu_{N}}\left(k, x_{0}\right)$ (solid) and optimal predictions $x_{u_{N, x(k)}^{*}}(\cdot, x(k))$ (dashed) along $x(k)$ for Example 3.1 with $N=5, x=0.5$ and $\mathbb{X}=[-0.5,0.5]$

When increasing $N$, the closed loop solution ends up in increasingly smaller neighborhoods of $x^{e}$, whose diameter actually shrinks down exponentially. This exponential decay is also reflected in the infinite horizon averaged value $J_{\infty}^{\text {cl }}\left(x, \mu_{N}\right)$, which converges to the optimal value $V_{\infty}(x)=0$ exponentially fast. Figure 2 illustrates this phenomenon and also shows that for the admissible set $\mathbb{X}=[-0.5,0.5]$ the values $J_{\infty}^{c l}\left(x, \mu_{N}\right)$ are smaller - and thus better - than for the larger set $\mathbb{X}=[-1,1]$.

Example 3.2 The second example is a linearized continuously stirred tank reactor model taken from [6] with 


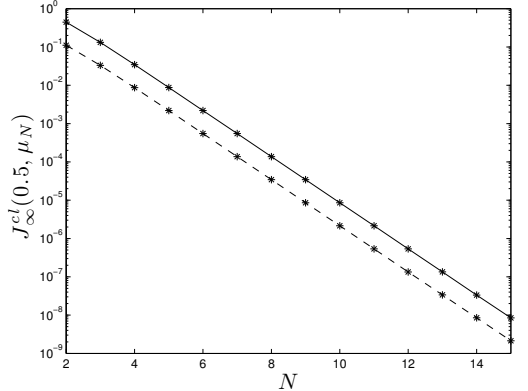

Fig. 2. $J_{\infty}^{c l}\left(x, \mu_{N}\right)$ for Example 3.1 with $N=2, \ldots, 15$, $x=0.5, \mathbb{X}=[1,1]$ (solid) and $\mathbb{X}=[-0.5,0.5]$ (dashed)

two dimensional affine linear dynamics

$$
\begin{aligned}
x(k+1)= & \left(\begin{array}{ll}
0.8353 & 0 \\
0.1065 & 0.9418
\end{array}\right) x(k) \\
& +\left(\begin{array}{r}
0.00457 \\
-0.00457
\end{array}\right) u(k)+\left(\begin{array}{l}
0.5559 \\
0.5033
\end{array}\right)
\end{aligned}
$$

and stage cost $\ell(x, u)=\|x\|^{2}+0.05 u^{2}$. We use the state and control constraints $\mathbb{X}=[-100,100]^{2}$ and $\mathbb{U}=$ $[-10,10]$. Among all the steady states of the dynamics, the point $x^{e} \approx(3.546,14.653)^{T}$ with $u^{e} \approx 6.163$ is the one with the lowest cost $\ell^{e}:=\ell\left(x^{e}, u^{e}\right) \approx 229.1876$. Observe that $x=0, u=0$ where the cost function attains its minimum $\ell(0,0)=0$ is not an equilibrium of the $d y$ namics.

The solutions exhibit a similar behavior as for Example 3.1: the open loop optimal trajectories first move towards $x^{e}$ and then move away while the closed loop trajectories converge to an equilibrium close to $x^{e}$ (Figure $3)$ and the closed loop performance $J_{\infty}^{c l}\left(x, \mu_{N}\right)$ converges exponentially towards $\ell^{e}$ for $N \rightarrow \infty$ (Figure 4 ).

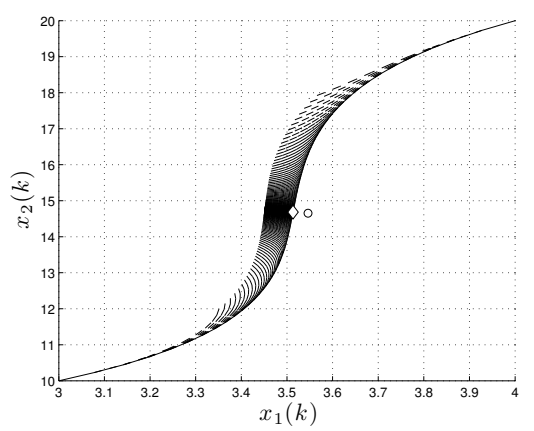

Fig. 3. Phase space plot of two closed loop trajectories $x(k)=x_{\mu_{N}}\left(k, x_{0}\right)$ (solid) and optimal predictions $x_{u_{N, x(k)}^{*}}(\cdot, x(k))$ (dashed) along $x(k)$ for Example 3.2 with $N=10$ and $x_{0}=(4,20)^{T}$ and $x_{0}=(3,10)^{T}$. The diamond indicates the equilibrium of the closed loop dynamics and the circle indicates the optimal steady state.

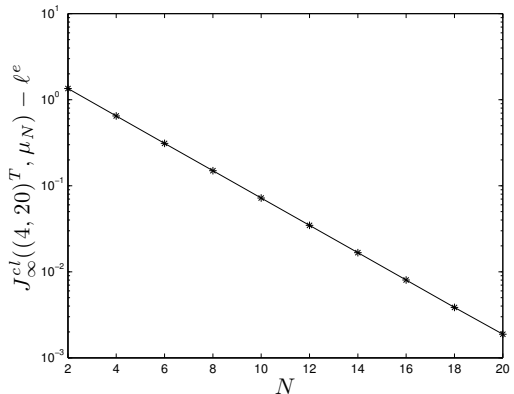

Fig. 4. $J_{\infty}^{c l}\left(x, \mu_{N}\right)-\ell^{e}$ for Example 3.2 with $N=2, \ldots, 20$ and $x=(4,20)^{T}$

\section{Value convergence}

Our goal in this section is to investigate the dependence of $J_{\infty}^{c l}\left(x, \mu_{N}\right)$ on $N$. The following Proposition 4.1 gives an upper bound for this value. Its proof uses the classical receding horizon proof technique to prolong a suitable control sequence of length $N$ in order to obtain a sequence of length $N+1$ for which the difference between $J_{N+1}$ and $V_{N}$ can be estimated. However, since the optimal trajectories for the finite horizon problem end up far away from $x^{e}$, in the setting considered in this paper it is in general not efficient to construct a suitable prolonged control sequence by adding an additional element at the end. Instead, we use control sequences in which an additional element is inserted at an arbitrary place into the control sequence. In Proposition 4.1 we assume that a suitably extended control sequence with an additional element inserted at time $k_{N, x}$ has already been constructed and formulate conditions on this sequence under which we can derive estimates on $J_{\infty}^{c l}\left(x, \mu_{N}\right)$. Sufficient conditions under which such a sequence can be constructed will then be introduced in the subsequent Theorem 4.2. In order to facilitate this construction, in Proposition 4.1 we do not assume optimality, but rather only approximate optimality with a suitable bound on the error term.

Proposition 4.1 Assume there are $N_{0}>0$ and $\delta_{1}, \delta_{2} \in$ $\mathcal{L}_{\mathbb{N}}$ such that for each $x \in \mathbb{X}$ and $N \geq N_{0}$ there exists a control sequence $u_{N, x} \in \mathbb{U}^{N+1}$ and $\bar{k}_{N, x} \in\{0, \ldots, N\}$ satisfying the following conditions.

(i) The inequality $J_{N}^{\prime}(x) \leq V_{N}(x)+\delta_{1}(N) / N$ holds for

$$
J_{N}^{\prime}(x):=\frac{1}{N} \sum_{\substack{k=0 \\ k \neq k_{N, x}}}^{N} \ell\left(x_{u_{N, x}}(k, x), u_{N, x}(k)\right)
$$

(ii) There exists $\ell_{0} \in \mathbb{R}$ such that for all $x \in \mathbb{X}$ the inequality $\ell\left(x_{u_{N, x}}\left(k_{N, x}, x\right), u_{N, x}\left(k_{N, x}\right)\right) \leq \ell_{0}+\delta_{2}(N)$ holds. 
Then the inequalities

$$
\begin{aligned}
J_{K}^{c l}\left(x, \mu_{N}\right) \leq & \frac{N}{K} V_{N}(x)-\frac{N}{K} V_{N}\left(x_{\mu_{N}}(K)\right) \\
& +\ell_{0}+\delta_{1}(N-1)+\delta_{2}(N-1)
\end{aligned}
$$

and

$$
J_{\infty}^{c l}\left(x, \mu_{N}\right) \leq \ell_{0}+\delta_{1}(N-1)+\delta_{2}(N-1)
$$

hold for all $x \in \mathbb{X}$, all $N \geq N_{0}+1$ and all $K \in \mathbb{N}$.

Proof: Fix $x \in \mathbb{X}$ and $N \geq N_{0}+1$. Abbreviating $x(k)=x_{\mu_{N}}(k, x)$, from (3) for any $k \geq 0$ we get $\ell\left(x(k), \mu_{N}(x(k))\right) / K=N V_{N}(x(k)) / K-(N-$ 1) $V_{N-1}(x(k+1)) / K$. Summing up for $k=0, \ldots, K-1$ then yields

$$
\begin{aligned}
& J_{K}^{c l}\left(x, \mu_{N}\right)=\frac{1}{K} \sum_{k=0}^{K-1} \ell\left(x(k), \mu_{N}(x(k))\right) \\
&=\frac{N}{K} V_{N}(x(0))-\frac{N-1}{K} V_{N-1}(x(K)) \\
&+\frac{1}{K} \sum_{k=1}^{K-1}\left(N V_{N}(x(k))-(N-1) V_{N-1}(x(k))\right) .
\end{aligned}
$$

Now we investigate the terms in (6). Property (i) with $N-1$ in place of $N$ and $x=x(k)$ implies $(N-1) V_{N-1}(x(k)) \geq(N-1) J_{N-1}^{\prime}(x(k))-\delta_{1}(N-1)$. Furthermore, by optimality of $V_{N}$ we get $V_{N}(x(k)) \leq$ $J_{N}\left(x(k), u_{N-1, x(k)}\right)$. Combining these inequalities, using the definition of $J_{N}$ and $J_{N}^{\prime}$ and (ii), for the summands of (6) we get

$$
\begin{aligned}
& N V_{N}(x(k))-(N-1) V_{N-1}(x(k)) \\
& \leq \underbrace{N J_{N}\left(x(k), u_{N-1, x(k)}\right)-(N-1) J_{N-1}^{\prime}(x(k))} \\
& \quad+\delta_{1}(N-1) \\
& \leq \ell_{0}+\delta_{2}(N-1)+\delta_{1}(N-1) .
\end{aligned}
$$

Recalling that $x(0)=x$ and inserting (7) for $k=$ $1, \ldots, K-1$ into $(6)$ yields $J_{K}^{c l}\left(x, \mu_{N}\right) \leq N V_{N}(x) / K-$ $(N-1) V_{N-1}(x(K)) / K+(K-1)\left(\ell_{0}+\delta_{2}(N-1)+\right.$ $\left.\delta_{1}(N-1)\right) / K$. Using (7) for $k=K$ and dividing by $K$ furthermore yields $-(N-1) V_{N-1}(x(K)) / K \leq$ $-N V_{N}(x(K)) / K+\left(\ell_{0}+\delta_{2}(N-1)+\delta_{1}(N-1)\right) / K$. Thus, we get (4). Inequality (5) follows from (4) by letting $K \rightarrow \infty$ since $V_{N}(x(K)) \geq \ell_{\min }$.

In order to apply Proposition 4.1, we need to construct the control sequences $u_{N, x}$ meeting its assumptions. The following theorem gives conditions on the finite horizon optimal value functions and trajectories under which such a construction is possible. Its statement is constructive in the sense that $u_{N, x}$ is explicitly constructed in the proof and its conditions can be rigorously checked for our motivating examples, as shown in the subsequent sections.

Theorem 4.2 Assume that there exists a set $\mathbb{Y} \subseteq \mathbb{X}$ and a value $\ell_{0} \geq 0$ such that for each $x \in \mathbb{Y}$ there is a control value $u \in \mathbb{U}$ with $f(x, u) \in \mathbb{Y}$ and $\ell(x, u) \leq \ell_{0}$. Assume furthermore that there exist $\bar{\delta}>0$ such that the following properties hold.

(a) There exists $\gamma_{f}, \gamma_{\ell} \in \mathcal{K}_{\infty}$ such that for all $\delta \in(0, \bar{\delta}]$ and all $x \in \mathcal{B}_{\delta}(\mathbb{Y})$ there is $u_{x} \in \mathbb{U}$ such that $f\left(x, u_{x}\right) \in \mathbb{X}$ and the inequalities $\left|f\left(x, u_{x}\right)\right|_{\mathbb{Y}} \leq \gamma_{f}(\delta)$ and $\ell\left(x, u_{x}\right) \leq$ $\ell_{0}+\gamma_{\ell}(\delta)$ hold.

(b) There exists $N_{0} \in \mathbb{N}_{0}$ and $\gamma_{V} \in \mathcal{K}_{\infty}$ such that for all $\delta \in(0, \bar{\delta}]$, all $N \in \mathbb{N}$ with $N \geq N_{0}$ and all $x \in \mathcal{B}_{\delta}(\mathbb{Y})$ and $y \in \mathbb{Y}$ the inequality $\left|V_{N}(x)-V_{N}(y)\right| \leq \gamma_{V}(\delta) / N$ holds.

(c) There exists $\sigma \in \mathcal{L}_{\mathbb{N}}$ and $N_{1} \in \mathbb{N}$ with $N_{1} \geq N_{0}$ for $N_{0} \in \mathbb{N}_{0}$ from (b), such that for each $x \in \mathbb{X}$ and each $N \geq N_{1}$ there exists an optimal trajectory $x_{u_{N, x}^{*}}(\cdot, x)$ satisfying $\left|x_{u_{N, x}^{*}}\left(k_{x}, x\right)\right|_{\mathbb{Y}} \leq \sigma(N)$ for some $k_{x} \in\left\{0, \ldots, N-N_{0}\right\}$.

Then there exists $N_{2} \in \mathbb{N}$ such that the inequalities

$$
\begin{array}{r}
J_{K}^{c l}\left(x, \mu_{N}\right) \leq \frac{N}{K}\left(V_{N}(x)-V_{N}\left(x_{\mu_{N}}(K)\right)\right)+\ell_{0} \\
+\varepsilon(N-1)
\end{array}
$$

and

$$
J_{\infty}^{c l}\left(x, \mu_{N}\right) \leq \ell_{0}+\varepsilon(N-1)
$$

hold for all $x \in \mathbb{X}, K \in \mathbb{N}$, all $N \geq N_{2}+1$ and $\varepsilon \in \mathcal{L}_{\mathbb{N}}$ given by $\varepsilon(N)=\gamma_{V}(\sigma(N))+\gamma_{V}\left(\gamma_{f}(\sigma(N))\right)+\gamma_{\ell}(\sigma(N))$.

Proof: We show that the assumptions of Proposition 4.1 hold for $\delta_{1}(N)=\gamma_{V}(\sigma(N))+\gamma_{V}\left(\gamma_{f}(\sigma(N))\right)$ and $\delta_{2}(N)=\gamma_{\ell}(\sigma(N))$ and then use this theorem in order to conclude the assertion. Note that $\delta_{1}, \delta_{2} \in \mathcal{L}_{\mathbb{N}}$ and thus also $\varepsilon \in \mathcal{L}_{\mathbb{N}}$.

To establish the assumptions of Proposition 4.1, we choose $N_{2} \geq N_{1}$ such that $\sigma\left(N_{2}\right) \leq \bar{\delta}$ and $\gamma_{f}\left(\sigma\left(N_{2}\right)\right) \leq \bar{\delta}$ holds for $\sigma$ from (c) and $\gamma_{f}$ from (a). Now pick $N \geq N_{2}$, $x \in \mathbb{X}$ and the corresponding optimal control $u_{N, x}^{*} \in$ $\mathbb{U}^{N}(x)$ from (c). Let $k_{x}$ be the time index from (c), abbreviate $x^{\prime}=x_{u_{N, x}^{*}}\left(k_{x}, x\right)$ and let $u_{x^{\prime}}$ be the control value from (a) for $x=x^{\prime}$. Let $x^{\prime \prime}=f\left(x^{\prime}, u_{x^{\prime}}\right)$ and let $u_{N-k_{x}, x^{\prime \prime}}^{*}$ be an optimal control sequence for initial value $x=x^{\prime \prime}$ and horizon $N-k_{x}$. Using these values, we define the control sequence $u_{N, x} \in \mathbb{U}^{N+1}(x)$ by $u_{N, x}(k):=u_{N, x}^{*}(k)$ for $k=0, \ldots, k_{x}-1, u_{N, x}(k):=u_{x^{\prime}}$ for $k=k_{x}$ and $u_{N, x}(k):=u_{N-k_{x}, x^{\prime \prime}}^{*}\left(k-k_{x}-1\right)$ for $k=k_{x}+1, \ldots, N$. This implies $x_{u_{N, x}}(k, x)=x_{u_{N, x}^{*}}(k, x)$ for $k=0, \ldots, k_{x}$,

$$
\left|x^{\prime}\right|_{\mathbb{Y}} \leq \sigma(N), \quad\left|x^{\prime \prime}\right|_{\mathbb{Y}}=\left|f\left(x^{\prime}, u_{x^{\prime}}\right)\right|_{\mathbb{Y}} \leq \gamma_{f}(\sigma(N))
$$

and

$$
\ell\left(x^{\prime}, u_{x^{\prime}}\right) \leq \ell_{0}+\gamma_{\ell}(\sigma(N)) \text {. }
$$


Using the fact that (b) implies $V_{N}(y)=V_{N}\left(y^{\prime}\right)$ for all $y, y^{\prime} \in \mathbb{Y}$, from (10) and (b) it follows that we can pick an arbitrary $y \in \mathbb{Y}$ in order to conclude the inequality

$$
\begin{aligned}
V_{K}\left(x^{\prime \prime}\right) & \leq V_{K}(y)+\frac{\gamma_{V}\left(\gamma_{f}(\sigma(N))\right)}{K} \\
& \leq V_{K}\left(x^{\prime}\right)+\frac{\gamma_{V}(\sigma(N))+\gamma_{V}\left(\gamma_{f}(\sigma(N))\right)}{K} \\
& =V_{K}\left(x^{\prime}\right)+\frac{\delta_{1}(N)}{K}
\end{aligned}
$$

for any $K \in \mathbb{N}$ with $K \geq N_{0}$. By (c) we have that $K=N-k_{x} \geq N_{0}$. Now we distinguish two cases:

In case $N-k_{x} \geq 1$ we can use (12) with $K=N-k_{x} \geq$ $N_{0}$ in order to obtain

$$
\begin{aligned}
& \frac{1}{N-k_{x}} \sum_{k=k_{x}+1}^{N} \ell\left(x_{u_{N, x}}(k, x), u_{N, x}(k)\right) \\
& =J_{N-k_{x}}\left(x^{\prime \prime}, u_{N-k_{x}, x^{\prime \prime}}^{*}\right)=V_{N-k_{x}}\left(x^{\prime \prime}\right) \\
& \leq V_{N-k_{x}}\left(x^{\prime}\right)+\frac{\delta_{1}(N)}{N-k_{x}} .
\end{aligned}
$$

Setting $k_{x, N}=k_{x}$ in Proposition 4.1(i) we obtain

$$
\begin{aligned}
J_{N}^{\prime}(x)= & \frac{1}{N} \sum_{k=0}^{k_{x}-1} \ell\left(x_{u_{N, x}^{*}}(k), u_{N, x}^{*}(k)\right) \\
& +\frac{1}{N} \sum_{k=k_{x}+1}^{N} \ell\left(x_{u_{N, x}}(k), u_{N, x}(k)\right) \\
\leq & V_{N}(x)-\frac{N-k_{x}}{N} V_{N-k_{x}}\left(x^{\prime}\right) \\
& +\frac{N-k_{x}}{N}\left(V_{N-k_{x}}\left(x^{\prime}\right)+\frac{\delta_{1}(N)}{N-k_{x}}\right) \\
= & V_{N}(x)+\delta_{1}(N) / N,
\end{aligned}
$$

where we have used (2) and (13) in the second step. This shows Assumption (i) of Proposition 4.1 with $\delta_{1}(N)=\gamma_{V}\left(\sigma_{N}\right)+\gamma_{V}\left(\gamma_{f}(\sigma(N))\right)$.

In case $N-k_{x}=0$ we obtain $J_{N}^{\prime}(x)=V_{N}(x)$ and thus Assumption (i) of Proposition 4.1 holds with arbitrary $\delta_{1}(N)$. Hence, in both cases Assumption (i) of Proposition 4.1 holds with $\delta_{1}(N)=$ $\gamma_{V}\left(\sigma_{N}\right)+\gamma_{V}\left(\gamma_{f}(\sigma(N))\right)$.

Furthermore, from (11) we get the inequality $\ell\left(x_{u_{N, x}}\left(k_{x}, x\right), u_{N, x}\left(k_{x}\right)\right)=\ell\left(x^{\prime}, u_{x^{\prime}}\right) \leq \ell_{0}+\gamma_{\ell}(\sigma(N))$, i.e., Assumption (ii) of Proposition 4.1 with $\delta_{2}(N)=$ $\gamma_{\ell}(\sigma(N))$. Thus, Proposition 4.1 applies and (8) and (9) follow with $\varepsilon(N)=\delta_{1}(N)+\delta_{2}(N)=$ $\gamma_{V}(\sigma(N))+\gamma_{V}\left(\gamma_{f}(\sigma(N))\right)+\gamma_{\ell}(\sigma(N))$.

While Condition (a) from Theorem 4.2 is quite easy to check using continuity of $f$ and $\ell$, Conditions (b) and (c) are much more difficult to verify. In the next two sections we will thus discuss checkable sufficient conditions for Conditions (b) and (c). We start with Condition (c).

\section{Optimal steady states and the turnpike prop- erty}

Condition (c) demands that the optimal solution "passes by" near the set $\mathbb{Y}$. In this section we investigate this property for the special case where $\mathbb{Y}=\left\{x^{e}\right\}$ is an equilibrium. We derive a checkable sufficient condition based on the so called turnpike property and an asymptotic controllability condition. We start with the following definition of infinite horizon optimality of an equilibrium.

Definition 5.1 A pair $\left(x^{e}, u^{e}\right) \in \mathbb{X} \times \mathbb{U}$ is called an equilibrium or steady state if $f\left(x^{e}, u^{e}\right)=x^{e}$ holds. For a given steady state and stage cost $\ell$ we say that the system is optimally operated at steady state if for each initial value $x \in \mathbb{X}$ and each admissible control sequence $u \in$ $\mathbb{U}^{\infty}(x)$ the inequality $\liminf \operatorname{in}_{N \rightarrow \infty} J_{N}(x, u) \geq \ell\left(x^{e}, u^{e}\right)$ holds.

A sufficient condition for this property is obtained by the following procedure taken from [3]. We define a modified cost

$$
\tilde{\ell}(x, u):=\ell(x, u)+\lambda(x)-\lambda(f(x, u))
$$

for a given function $\lambda: \mathbb{X} \rightarrow \mathbb{R}$. Defining the averaged functional $\widetilde{J}_{N}(x, u):=\frac{1}{N} \sum_{k=0}^{N-1} \tilde{\ell}\left(x_{u}(k, x), u(k)\right)$, one obtains the identity

$$
\widetilde{J}_{N}(x, u)=J_{N}(x, u)+\frac{\lambda(x)}{N}-\frac{\lambda\left(x_{u}(N, x)\right)}{N} .
$$

Moreover, the inequality $\min _{x \in \mathbb{X}, u \in \mathbb{U}} \tilde{\ell}(x, u) \leq \tilde{\ell}\left(x^{e}, u^{e}\right)=$ $\ell\left(x^{e}, u^{e}\right)$ holds. Additionally, we make the following assumption.

Assumption 5.2 The function $\lambda$ in (14) is bounded on $\mathbb{X}$ and there exists a steady state $\left(x^{e}, u^{e}\right) \in \mathbb{X} \times \mathbb{U}$ and $\alpha_{\ell} \in$ $\mathcal{K}_{\infty}$ such that $\min _{u \in \mathbb{U}} \tilde{\ell}(x, u) \geq \ell\left(x^{e}, u^{e}\right)+\alpha_{\ell}\left(\left\|x-x^{e}\right\|\right)$ holds for all $x \in \mathbb{X}$ with $\tilde{\ell}$ from (14).

One checks that Assumption 5.2 is satisfied for Examples 3.1 and 3.2 for $\lambda(x)=-x^{2} / 2$ and $\lambda(x)=c^{T} x$ with $c^{T} \approx(-368.6684,-503.5415)^{T}$, respectively. More generally, as remarked in [6], the inequality in Assumption 5.2 always holds with a linear function $\lambda$ for linear control systems and strictly convex stage costs and convex constraints. Boundedness of $\lambda$ then follows if $\mathbb{X}$ is bounded.

A straightforward adaptation of [3, Theorem 2] shows that Assumption 5.2 implies that the system is optimally operated at steady state. Moreover, if the system is optimally operated at steady state and if the assumptions of Theorem 4.2 hold for $\mathbb{Y}=\left\{x^{e}\right\}$, then $\ell_{0}=\ell\left(x^{e}, u^{e}\right)$ and the convergence $\lim _{N \rightarrow \infty} J_{\infty}^{c l}\left(x, \mu_{N}\right)=V_{\infty}(x)$ follows.

The following theorem shows a consequence from Assumption 5.2 known as the turnpike property, cf. [5, Section 4.4]. Here we present it in a discrete time version and provide a quantitative estimate for the value $Q_{\varepsilon}$. 
Theorem 5.3 Assume that there exists $\lambda: \mathbb{X} \rightarrow \mathbb{R}$ satisfying Assumption 5.2. Then for each $x \in \mathbb{X}$, each $\delta>0$, each control sequence $u \in \mathbb{U}^{N}(x)$ satisfying $J(x, u) \leq$ $\ell\left(x^{e}, u^{e}\right)+\delta / N$ and each $\varepsilon>0$ the value $Q_{\varepsilon}:=\#\{k \in$ $\left.\{0, \ldots, N-1\} \mid\left\|x_{u}(k, x)-x^{e}\right\| \leq \varepsilon\right\}$ satisfies the inequality $Q_{\varepsilon} \geq N-(\delta+C) / \alpha_{\ell}(\varepsilon)$.

Proof: For $C:=2 \sup _{x \in \mathbb{X}}|\lambda(x)|<\infty,(15)$ implies

$$
\widetilde{J}_{N}(x, u) \leq J_{N}(x, u)+\frac{C}{N} \leq \ell\left(x^{e}, u^{e}\right)+\frac{\delta+C}{N} .
$$

Now assume that $Q_{\varepsilon}<N-(\delta+C) / \alpha_{\ell}(\varepsilon)$. This means that there exists a set $\mathcal{N} \subseteq\{0, \ldots, N-1\}$ of $N-Q_{\varepsilon}>(\delta+C) / \alpha_{\ell}(\varepsilon)$ times instants such that $\left\|x_{u}(k, x)-x^{e}\right\|>\varepsilon$ holds for all $k \in \mathcal{N}$. Using Assumption 5.2 this implies $\widetilde{J}_{N}(x, u) \geq(N-$ $\left.Q_{\varepsilon}\right)\left(\ell\left(x^{e}, u^{e}\right)+\alpha_{\ell}(\varepsilon)\right) / N+Q_{\varepsilon} \ell\left(x^{e}, u^{e}\right) / N=\ell\left(x^{e}, u^{e}\right)+$ $\left(N-Q_{\varepsilon}\right) \alpha_{\ell}(\varepsilon) / N>\ell\left(x^{e}, u^{e}\right)+(\delta+C) / N$. This contradicts (16) and thus proves the theorem.

The last ingredient we need in order to conclude Condition (c) of Theorem 4.2 from the turnpike property is an asymptotic controllability property with respect to the stage cost $\ell$ which we formulate after the following definition of a subclass of $\mathcal{K} \mathcal{L}$-functions.

Definition 5.4 By $\mathcal{K} \mathcal{L S}$ we denote the class of summable $\mathcal{K} \mathcal{L}$ functions which sum up to a $\mathcal{K}$ function, i.e., the class of functions $\beta \in \mathcal{K} \mathcal{L}$ for which $\sum_{k=0}^{\infty} \beta(r, k)$ is finite for all $r \geq 0$ and for which $\gamma_{\beta}: \mathbb{R}_{0}^{+} \rightarrow \mathbb{R}$ given by $\gamma_{\beta}(r):=\sum_{k=0}^{\infty} \beta(r, k)$ satisfies $\gamma_{\beta} \in \mathcal{K}$.

Assumption 5.5 There exists $\beta \in \mathcal{K} \mathcal{L} \mathcal{S}$ such that for each $x \in \mathbb{X}$ and each $N \in \mathbb{N}$ there is a control function $u \in \mathbb{U}^{N}(x)$ such that the inequality $\ell\left(x_{u}(k, x), u(k)\right) \leq$ $\ell\left(x^{e}, u^{e}\right)+\beta\left(\left\|x-x^{e}\right\|, k\right)$ holds for all $k=0, \ldots, N-1$.

With this property, which again holds for the examples from Section 3, we can now prove the main theorem of this section.

Theorem 5.6 Assume that there exists $\lambda: \mathbb{X} \rightarrow \mathbb{R}$ satisfying Assumption 5.2. Assume, moreover, that $\mathbb{X}$ is bounded and that Assumption 5.5 holds. Then Condition (c) of Theorem 4.2 holds for $\mathbb{Y}=\left\{x^{e}\right\}$.

Proof: The asymptotic controllability assumption yields $J_{N}(x, u) \leq \ell\left(x^{e}, u^{e}\right)+\gamma_{\beta}\left(\left\|x-x^{e}\right\|\right) / N$. Hence, since $\mathbb{X}$ and thus $\left\|x-x^{e}\right\|$ is bounded for all $x \in \mathbb{X}$ we get $V_{N}(x) \leq \ell\left(x^{e}, u^{e}\right)+\delta / N$ for $\delta=\gamma_{\beta}\left(\max _{x \in \mathbb{X}}\left(\left\|x-x^{e}\right\|\right)\right)$. Now we set $C:=2 \sup _{x \in \mathbb{X}}|\lambda(x)|<\infty$, choose $N_{1}=N_{0}$ and set $\sigma(N)$ arbitrary for $N \leq N_{1}$ and $\sigma(N):=\alpha_{\ell}^{-1}\left((\delta+C) /\left(N-N_{0}\right)\right)$ otherwise, with $\alpha_{\ell} \in \mathcal{K}_{\infty}$ from Assumption 5.2. Clearly, this function lies in $\mathcal{L}_{\mathbb{N}}$ because as $N \rightarrow \infty$ the argument of $\alpha_{\ell}^{-1}$ tends to 0 and thus $\alpha_{\ell}^{-1}$ does so, too, since inverse functions of $\mathcal{K}_{\infty}$ functions are again $\mathcal{K}_{\infty}$ functions. This choice of $\sigma$ implies $Q_{\sigma(N)} \geq N-(\delta+C) / \alpha_{\ell}(\sigma(N))=N_{0}$. Hence, there are at least $N_{0}$ time instants $k$ for which $\left\|x_{u}(k, x)-x^{e}\right\| \leq \sigma(N)$ holds and consequently at least one of these $k$ must satisfy $k \in\left\{0, \ldots, N-N_{0}\right\}$. Condition (c) thus holds if we choose $k_{x}$ as this $k$.

Every system which is exponentially stabilizable at $x^{e}$ by a locally Lipschitz feedback law satisfies Assumption 5.5 if $\ell$ is locally Lipschitz near $x^{e}$; particularly, this applies to our examples from Section 3. Together with the observation from [6] cited after Assumption 5.2 this implies that Theorem 5.6 holds for every exponentially stabilizable linear system with locally Lipschitz and strictly convex stage cost and convex constraints.

We conjecture that many of the results in this section can be extended to more general sets $\mathbb{Y}$. However, due to space restrictions we postpone this discussion to a separate paper. A nonlinear $2 \mathrm{~d}$ system where $\mathbb{Y}$ is a periodic orbit can be found in [7, Example 6].

\section{Controllability conditions}

After having derived sufficient conditions for Condition (c) of Theorem 4.2, we now do the same for Condition (b). To this end, we introduce the following assumption in which $\left(x^{e}, u^{e}\right)$ denotes the steady state from Assumption 5.2 .

Assumption 6.1 There exists $\delta_{c}>0, d \in \mathbb{N}$ and $\gamma_{x}, \gamma_{u}, \gamma_{c} \in \mathcal{K}_{\infty}$ such that for each trajectory $x_{u_{1}}(k, x)$ with $u_{1} \in \mathbb{U}^{d}(x)$ satisfying $x_{u}(k, x) \in \mathcal{B}_{\delta_{c}}\left(x^{e}\right)$ for all $k=0, \ldots, d$ and all $x_{1}, x_{2} \in \mathcal{B}_{\delta_{c}}\left(x^{e}\right)$ there exists $u_{2} \in \mathbb{U}^{d}(x)$ satisfying $x_{u_{2}}\left(d, x_{1}\right)=x_{2}$ and the estimates $\left\|x_{u_{2}}\left(k, x_{1}\right)-x_{u_{1}}(k, x)\right\| \leq \gamma_{x}\left(\max \left\{\| x_{1}-\right.\right.$ $\left.\left.x\|,\| x_{2}-x_{u_{1}}(d, x) \|\right\}\right),\left\|u_{2}(k)-u_{1}(k)\right\| \leq \gamma_{u}\left(\max \left\{\| x_{1}-\right.\right.$ $\left.\left.x\|,\| x_{2}-x_{u_{1}}(d, x) \|\right\}\right)$ and $\mid \ell\left(x_{u_{2}}\left(k, x_{1}\right), u_{2}(k)\right)-$ $\ell\left(x_{u_{1}}(k, x), u_{1}(k)\right) \mid \leq \gamma_{c}\left(\max \left\{\left\|x_{1}-x\right\|,\left\|x_{2}-x_{u_{1}}(d, x)\right\|\right\}\right)$ for all $k=0, \ldots, d-1$.

This assumption holds for all controllable linear systems in $\mathbb{R}^{n}$ with $d=n$, provided $x^{e}$ and $u^{e}$ are in the interior of the constraint sets $\mathbb{X}$ and $\mathbb{U}$. It is thus satisfied for both examples from Section 3 for $d=1$. More generally, we conjecture that for systems with $\mathbb{X} \subseteq \mathbb{R}^{n}$ and $\mathbb{U} \subseteq \mathbb{R}^{m}$ we can conclude Assumption 6.1 if the linearization of $f$ in $\left(x^{e}, u^{e}\right)$ is controllable and the trajectory $x_{u_{1}}(k, x)$ and the values $u_{1}(k)$ lie in the interior of $\mathbb{X}$ and $\mathbb{U}$, respectively.

The following lemma shows an important consequence from Assumption 6.1 and the turnpike property under the following assumption.

Assumption 6.2 There exists $\alpha_{u} \in \mathcal{K}_{\infty}$ such that the inequality $\tilde{\ell}(x, u) \leq \ell\left(x^{e}, u^{e}\right)+\alpha_{u}\left(\left\|x-x^{e}\right\|+\left\|u-u^{e}\right\|\right)$ holds for all $x \in \mathbb{X}$ and $u \in \mathbb{U}$. 
Since $\ell\left(x^{e}, u^{e}\right)=\tilde{\ell}\left(x^{e}, u^{e}\right)$, this assumption holds, e.g., if $\ell$ and $\lambda$ are Lipschitz, which is the case in our examples.

Lemma 6.3 Suppose that Assumptions 5.2, 6.1 and 6.2 hold. Then there exists $N_{1}>0$, a function $P: \mathbb{N} \rightarrow \mathbb{N}$ with $P(N) \geq N / 2$ and $\eta: \mathbb{N} \times \mathbb{R}_{0}^{+} \rightarrow \mathbb{R}_{0}^{+}$with $\eta(N, r) \rightarrow$ 0 if $N \rightarrow \infty$ and $r \rightarrow 0$ such that the open loop optimal trajectories with horizon $N \geq N_{1}$ starting in $x_{1} \in$ $\mathcal{B}_{\delta_{c}}\left(x_{e}\right)$ satisfy $\left\|x_{u_{N, x_{1}}^{*}}\left(k, x_{1}\right)-x^{e}\right\| \leq \eta\left(N,\left\|x_{1}-x^{e}\right\|\right)$ for all $k=0, \ldots, P(N)$.

Proof: Using Assumption 6.1 with $x_{1}$ from the assumption, $x=x_{2}=x^{e}$ and $u_{1} \equiv u^{e}$ we get $J_{d}\left(x_{1}, u_{2}\right) \leq$ $\ell\left(x^{e}, u^{e}\right)+\gamma_{c}\left(\left\|x_{1}-x^{e}\right\|\right)$ and $x_{u_{2}}\left(d, x_{1}\right)=x^{e}$. Picking $N \geq d$ and extending $u_{2}$ by setting $u_{2}(k)=u^{e}$ for $k=d, \ldots, N$ we thus obtain $J_{N}\left(x_{1}, u_{N, x_{1}}^{*}\right) \leq$ $J_{N}\left(x_{1}, u_{2}\right) \leq \ell\left(x^{e}, u^{e}\right)+d \gamma_{c}\left(\left\|x_{1}-x^{e}\right\|\right) / N$. Hence, we can apply Theorem 5.3 to $x=x_{1}$ and $u=u_{2}$ with $\delta=d \gamma_{c}\left(\left\|x_{1}-x^{e}\right\|\right)$ and we pick $\varepsilon>0$ and $N \in \mathbb{N}$ such that $Q_{\varepsilon} \geq 2 d$ holds. We set $P(N)$ to be the largest $k$ such that $\left\|x_{u_{N, x_{1}}^{*}}\left(k, x_{1}\right)-x^{e}\right\| \leq \varepsilon$ holds. With this choice, $P(N) \geq Q_{\varepsilon} \geq 2 d$ holds. Now we use Assumption 6.1 with $x_{1}=x=x^{e}, u_{1} \equiv u^{e}$ and $x_{2}=x_{u_{N, x_{1}}^{*}}\left(P(N), x_{1}\right)$ and denote the resulting control sequence by $\bar{u}_{2}$. This sequence satisfies $J_{d}\left(x^{e}, \bar{u}_{2}\right) \leq \ell\left(x^{e}, u^{e}\right)+\gamma_{c}(\varepsilon)$.

Using $u_{2}$ from above we now define a new control sequence $\bar{u}$ via $\bar{u}(k)=u_{2}(k)$ for $k=0, \ldots, d-1, \bar{u}(k)=u^{e}$ for $k=d, \ldots, P(N)-d-1, \bar{u}(k)=u_{2}(k-P(N)+d)$ for $k=P(N)-d, \ldots, P(N)-1$ and $\bar{u}(k)=u_{N, x_{1}}^{*}(k)$ for $k=P(N), \ldots, N-1$. By construction of $\bar{u}$ we get $x_{\bar{u}}\left(k, x_{1}\right)=x_{u_{2}}\left(k, x_{1}\right)$ for $k=0, \ldots, d$, $x_{\bar{u}}\left(k, x_{1}\right)=x^{e}$ for $k=d, \ldots, P(N)-d, x_{\bar{u}}\left(k, x_{1}\right)=$ $x_{u_{2}}\left(k-P(N)+d, x^{e}\right)$ for $k=P(N)-d, \ldots, P(N)$ and $x_{\bar{u}}\left(k, x_{1}\right)=x_{u_{N, x_{1}}^{*}}\left(k, x_{1}\right)$ for $k=P(N), \ldots, N$.

Since by the optimality principle the last piece of the trajectory is optimal for horizon $N-P(N)$, we obtain $J_{P(N)}\left(u_{N, x_{1}}^{*}, x_{1}\right) \leq J_{P(N)}\left(\bar{u}, x_{1}\right)$. Moreover, since $x_{\bar{u}}\left(P(N), x_{1}\right)=x_{u_{N, x_{1}}^{*}}\left(P(N), x_{1}\right)$, by (15) we get $J_{N}\left(u_{N, x_{1}}^{*}, x_{1}\right)-J_{P(N)}\left(\bar{u}, x_{1}\right)=\widetilde{J}_{N}\left(u_{N, x_{1}}^{*}, x_{1}\right)-$ $\widetilde{J}_{P(N)}\left(\bar{u}, x_{1}\right)$ and thus

$$
\widetilde{J}_{P(N)}\left(u_{N, x_{1}}^{*}, x_{1}\right) \leq \widetilde{J}_{P(N)}\left(\bar{u}, x_{1}\right) .
$$

From the construction of $\bar{u}$ via Assumption 6.1 we now get the estimates $x_{\bar{u}}\left(k, x_{1}\right)-x^{e} \| \leq \gamma_{x}\left(\left\|x_{1}-x^{e}\right\|\right)$ and $\left\|\bar{u}(k)-u^{e}\right\| \leq \gamma_{u}\left(\left\|x_{1}-x^{e}\right\|\right)$ for $\bar{k}=0, \ldots, d-1$ and $\left\|x_{\bar{u}}\left(k, x_{1}\right)-x^{e}\right\| \leq \gamma_{x}(\varepsilon)$ and $\left\|\bar{u}(k)-u^{e}\right\| \leq \gamma_{u}(\varepsilon)$ for $k=$ $P(N)-d+1, \ldots, P(N)$. For $k=d, \ldots, P(N)-d$ we get $x_{\bar{u}}\left(k, x_{1}\right)=x^{e}$ and $\bar{u}(k)=u^{e}$. Using Assumption 6.2, for the modified functional this implies $\widetilde{J}_{P(N)}\left(\bar{u}, x_{1}\right) \leq$ $\ell\left(x^{e}, u^{e}\right)+\frac{d}{P(N)} \alpha_{u}\left(\gamma_{x}\left(\left\|x_{1}-x^{e}\right\|\right)+\gamma_{u}\left(\left\|x_{1}-x^{e}\right\|\right)\right)+$ $\frac{d}{P(N)} \alpha_{u}\left(\gamma_{x}(\varepsilon)+\gamma_{u}(\varepsilon)\right)$. On the other hand, if we assume that $\left\|x_{u_{N, x_{1}}^{*}}\left(k, x_{1}\right)-x^{e}\right\| \geq \Delta$ for some $\Delta>0$ and some $k \in\{0, \ldots, P(N)-1\}$, then from Assumption 5.2 we get $\widetilde{J}_{P(N)}\left(x_{1}, u_{N, x_{1}}^{*}\right) \geq \ell\left(x^{e}, u^{e}\right)+\alpha_{\ell}(\Delta) / P(N)$. Hence, in case that $\Delta>\alpha_{\ell}^{-1}\left(d \alpha_{u}\left(\gamma_{x}\left(\left\|x_{1}-x^{e}\right\|\right)+\gamma_{u}\left(\| x_{1}-\right.\right.\right.$ $\left.\left.\left.x^{e} \|\right)\right)+d \alpha_{u}\left(\gamma_{x}(\varepsilon)+\gamma_{u}(\varepsilon)\right)\right)$ we get the inequality $\widetilde{J}_{P(N)}\left(x_{1}, u_{N, x_{1}}^{*}\right)>\widetilde{J}_{P(N)}\left(\bar{u}, x_{1}\right)$ which contradicts $(17)$. Thus, we get $\Delta \leq \alpha_{\ell}^{-1}\left(d \alpha_{u}\left(\gamma_{x}\left(\left\|x_{1}-x^{e}\right\|\right)+\gamma_{u}\left(\| x_{1}-\right.\right.\right.$ $\left.\left.\left.x^{e} \|\right)\right)+d \alpha_{u}\left(\gamma_{x}(\varepsilon)+\gamma_{u}(\varepsilon)\right)\right)$.

The assertion now follows by choosing $\varepsilon=\alpha_{\ell}^{-1}(2(\delta+$ $C) / N$ ) which implies $P(N) \geq Q_{\varepsilon} \geq N / 2$ as well as $\varepsilon \rightarrow 0$ as $N \rightarrow \infty$. Setting $N_{1}=4 d$ then ensures $P(N) \geq 2 d$ for $N \geq N_{1}$ and defining $\left.\eta(N, r):=\alpha_{\ell}^{-1}\left(d \alpha_{u}\left(\gamma_{x}(r)+\gamma_{u}(r)\right)\right)+d \alpha_{u}\left(\gamma_{x}(\varepsilon)+\gamma_{u}(\varepsilon)\right)\right)$ finally shows the assertion.

Using Lemma 6.3 we can now prove the following sufficient condition for Theorem 4.2(b).

Theorem 6.4 Under the Assumptions 5.2, 6.1 and 6.2, Condition (b) of Theorem 4.2 holds for $\mathbb{Y}=\left\{x^{e}\right\}$.

Proof: We choose $N_{0} \geq N_{1}$ and $\bar{\delta}>0$ such that $\bar{\delta} \leq \delta_{c}$ for the values from Lemma 6.3 and Assumption 6.1 holds and such that $\eta(N, r)<\delta_{c}$ in Lemma 6.3 holds for all $N \geq N_{0}$ and $r \in(0, \bar{\delta})$. We show the inequality in Condition (b) for all $x, y \in \mathcal{B}_{\bar{\delta}}\left(x^{e}\right)$ which particularly implies the assertion for $y=x^{e}$.

By Lemma 6.3 and since $\eta(N, \bar{\delta}) \leq \delta_{c}$ and $P(N) \geq d$ (cf. the construction in the proof of Lemma $6.3)$, the optimal trajectory starting in $x$ satisfies $x_{u_{N, x}^{*}}(k, x) \in \mathcal{B}_{\delta_{c}}\left(x^{e}\right)$ for $k=0, \ldots, d$. Thus, we can apply Assumption 6.1 with this $x, x_{1}=y, u_{1}=u_{N, x}^{*}$ and $x_{2}=x_{u_{N, x}^{*}}(d, x)$ in order to conclude that there exists $u_{2} \in \mathbb{U}^{d}(y)$ such that $x_{u_{2}}(d, y)=x_{u_{N, x}^{*}}(d, x)$ and $\ell\left(x_{u_{2}}(k, y), u_{2}(k)\right) \leq \ell\left(x_{u_{N, x}^{*}}(k, x), u_{N, x}^{*}(k)\right) \leq$ $\gamma_{c}(\|y-x\|)$ (note that $\left\|x_{2}-x_{u_{1}}(d, x)\right\|=0$ by choice of $\left.x_{2}\right)$. Extending $u_{2}$ via $u_{2}(k):=u_{N, x}^{*}(k)$ for $d, \ldots, N-1$ then yields

$$
\begin{aligned}
V_{N}(y) \leq & J_{N}\left(y, u_{2}\right) \leq \frac{1}{N} \sum_{k=0}^{N-1} \ell\left(x_{u_{N, x}^{*}}(k, x), u_{N, x}^{*}(k)\right) \\
+ & \frac{d}{N} \gamma_{c}(\|y-x\|)=V_{N}(x)+\frac{d}{N} \gamma_{c}(\|y-x\|)
\end{aligned}
$$

Setting $\gamma_{V}(r)=d \gamma_{c}(\|y-x\|)$ we thus obtain $V_{N}(y) \leq$ $V_{N}(x)+\gamma_{V}(\|x-y\|) / N$ and by exchanging $x$ and $y$ we get the converse inequality which shows Condition (b) of Theorem 4.2 .

Remark 6.5 The discussions after Theorem 5.6 and Assumption 6.1 imply that the assumptions of Theorem 5.6 and 6.4 hold for each controllable linear system with locally Lipschitz and strictly convex cost for which $\mathbb{U}$ is convex, $\mathbb{X}$ is convex and bounded and $x^{e}$ and $u^{e}$ lie in the interior of $\mathbb{X}$ and $\mathbb{U}$. Hence, Theorem 4.2 applies to all such systems. Note, however, that Example 3.1 shows that strict convexity of $\ell$ is not necessary. An in depth 
analysis of our assumptions for linear systems will be addressed in a separate paper.

Note that Figures 2 and 4 indicate that the error term $\varepsilon(N-1)$ in Theorem 4.2 decays exponentially as $N \rightarrow \infty$. Further numerical simulations revealed that also $\sigma(N)$ in Theorem 4.2(c) decays exponentially (from which, in turn, exponential convergence of $\varepsilon(N-1)$ can be concluded). In contrast to this, Theorem 5.6 only delivers linear convergence $\sigma(N) \rightarrow 0$. Conditions under which exponential convergence of $\sigma(N)$ can be guaranteed are currently under investigation.

\section{Trajectory convergence}

So far we have developed bounds for the values $J_{\infty}^{c l}\left(x, \mu_{N}\right)$ and $J_{N}^{c l}\left(x, \mu_{N}\right)$ along the closed loop trajectories. In this section, we investigate these trajectories, themselves, and give conditions under which convergence to a neighborhood of $\mathbb{Y}$ can be shown. Moreover, we discuss estimates on the transient performance of the closed loop trajectories. The results are formulated for general sets $\mathbb{Y}$ and for this purpose we will generalize some of the properties from the Sections 5 and 6 . We start our analysis with the following theorem.

Theorem 7.1 (i) Assume there are $N_{1} \in \mathbb{N}$ and $\delta \in \mathcal{L}_{\mathbb{N}}$ such that the inequality

$$
\begin{aligned}
& J_{K}^{c l}\left(x, \mu_{N}\right) \leq \\
& \quad \frac{N}{K}\left(V_{N}(x)-V_{N}\left(x_{\mu_{N}}(K)\right)\right)+\ell_{0}+\frac{\delta(N)}{\min \{N, K\}}
\end{aligned}
$$

holds for all $x \in \mathbb{X}$, all $N \geq N_{1}+1$ and all $K \in \mathbb{N}$. Assume furthermore that there exists a set $\mathbb{Y} \subset \mathbb{X}$ and $a$ function $\eta \in \mathcal{L}_{\mathbb{N}}$ such that for all $N \geq N_{1}$ the inequality

$$
V_{N}(x) \geq \ell_{0}+\alpha\left(|x|_{\mathbb{Y}}\right) / N
$$

holds for all $x \in \mathbb{X} \backslash \mathbb{Y}$ with $|x|_{\mathbb{Y}}>\eta(N)$ and some $\alpha \in \mathcal{K}_{\infty}$. Then for all $N \geq N_{1}+1$ and all $k \geq N$ the inequality $\left|x_{\mu_{N}}(k)\right|_{\mathbb{Y}} \leq \max \left\{\eta(N), \alpha^{-1}(\delta(N))\right\}$ holds.

(ii) If, moreover, for all $N \geq N_{1}$ the inequality

$$
V_{N}(x) \leq \ell_{0}+\bar{\alpha}\left(|x|_{\mathbb{Y}}\right) / N
$$

holds for some $\bar{\alpha} \in \mathcal{K}_{\infty}$, then for all $N \geq N_{1}+1$ the inequality $\left|x_{\mu_{N}}(k)\right|_{\mathbb{Y}} \leq \max \left\{\eta(N), \alpha^{-1}\left(\bar{\alpha}\left(|x|_{\mathbb{Y}}\right)+\delta(N)\right)\right\}$ holds for all $k \in\{1, \ldots, N-1\}$ and all $x \in \mathbb{X}$ with $V_{k}(x) \geq \ell_{0}$.

Proof: (i) We abbreviate $x(k)=x_{\mu_{N}}(k)$ and observe that for $k \geq N$ the identity $x(N, x(k-N))=x(k)$ holds. Then, for all $N \geq N_{1}+1$, all $k \geq N$ and all $x(0) \in \mathbb{X}$, Inequality (18) applied with $x=x(k-N)$ and $K=N$ yields $V_{N}(x(k)) \leq V_{N}(x(k-N))-$ $J_{N}^{c l}\left(x(k-N), \mu_{N}\right)+\ell_{0}+\delta(N) / N$. Together with
$V_{N}(x(k-N)) \leq J_{N}^{c l}\left(x(k-N), \mu_{N}\right)$, this yields $V_{N}(x(k)) \leq \ell_{0}+\delta(N) / N$. Hence, we either get $|x(k)|_{\mathbb{Y}} \leq$ $\eta(N)$ or $\alpha\left(|x(k)|_{\mathbb{Y}}\right) \leq N\left(V_{N}(x(k))-\ell_{0}\right) \leq \delta(N)$ which implies the assertion.

(ii) From (18) with $K=k \leq N$ we get $V_{N}(x(k)) \leq$ $V_{N}(x)-k J_{K}^{c l}\left(x, \mu_{N}\right) / N+\overline{\frac{k}{N}} \ell_{0}+\delta(N) / N$. Now $J_{K}^{c l}\left(x, \mu_{N}\right) \geq V_{K}(x) \geq \ell_{0}$ yields $V_{N}(x(k)) \leq V_{N}(x)-$ $k J_{K}^{c l}\left(x, \mu_{N}\right) / N+k \ell_{0} / N+\delta(N) / N \leq V_{N}(x)+\delta(N) / N$. Hence, we either get $|x(k)|_{\mathbb{Y}} \leq \eta(N)$ or $\alpha\left(|x(k)|_{\mathbb{Y}}\right) \leq$ $N\left(V_{N}(x(k))-\ell_{0}\right) \leq N\left(V_{N}(x)-\ell_{0}+\frac{1}{N} \delta(N)\right) \leq$ $\bar{\alpha}\left(|x|_{\mathbb{Y}}\right)+\delta(N)$ which implies the claim.

Note that Theorem 7.1(i) provides a bound for $k \geq N$ while Theorem 7.1(ii) yields a bound for $k=1, \ldots, N-$ 1. If both estimates hold, then one could also construct an upper bound of the form $\beta\left(|x|_{\mathbb{Y}}, k\right)+\varepsilon(N)$ as in more standard practical asymptotic stability estimates.

Based on the numerical observation that $\varepsilon(N-1)$ in (8) decays exponentially for the Examples 3.1 and 3.2, we can derive (18) from (8) with $\delta(N)=N \varepsilon(N-1)$. However, while the conditions (20) and (19) hold for Example 3.1 (see [7, Example 4]), neither of them is satisfied for Example 3.2.

A remedy for this problem can be obtained by considering the modified stage from Section 5. From Assumption 5.2 one easily concludes $\widetilde{V}_{N}(x) \geq \ell\left(x^{e}, u^{e}\right)+$ $\frac{1}{N} \alpha_{\ell}\left(\left\|x-x^{e}\right\|\right)$ and thus (19) and from Assumption 5.5 one can derive (20). Figure 5 illustrates how the optimal value functions change.

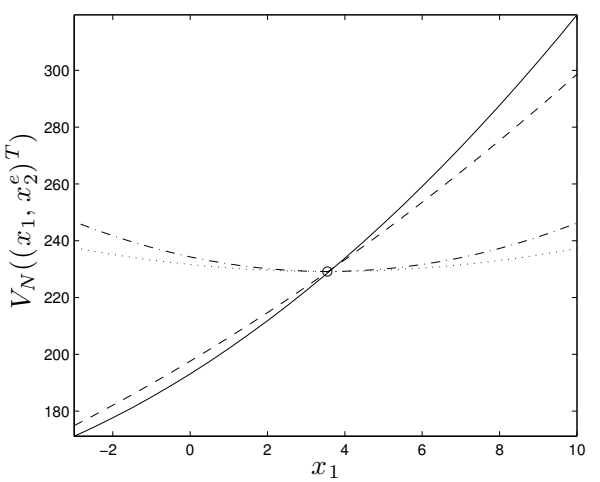

Fig. 5. Numerically computed $V_{N}\left(\left(\cdot, x_{2}^{e}\right)^{T}\right)$ for $N=10$ (solid) and $N=30$ (dashed) and $\widetilde{V}_{N}\left(\left(\cdot, x_{2}^{e}\right)^{T}\right)$ for $N=10$ (dashdotted) and $N=30$ (dotted) for Example 3.2. The circle indicates $\left(x_{1}^{e}, \ell_{0}\right)^{T}$.

Unfortunately, however, when passing from $\ell$ to $\tilde{\ell}$, the open loop optimal trajectories and thus also the closed loop trajectories change. The key to the fact that we can still use the modified problem in order to conclude convergence for the original problem lies in the fact that for suitable horizon $P \leq N$ the averaged functional yields (almost) the same value along the optimal trajectories $x_{u_{N, x}^{*}}(n, x)$ and $x_{\tilde{u}_{N, x}^{*}}(n, x)$. After providing two preliminary lemmas we will make this statement precise in 
Lemma 7.5, below. To this end, we assume the following version of the turnpike property.

Assumption 7.2 We assume that there exists $C^{\prime} \geq 0$ such that for each $x \in \mathbb{X}$, the optimal control sequence $u_{x, N}^{*} \in \mathbb{U}^{N}(x)$ and each $\varepsilon>0$ the value $Q_{\varepsilon}:=\# \mathcal{P}_{\varepsilon}$ with $\mathcal{P}_{\varepsilon}:=\left\{\left.k \in\{0, \ldots, N-1\}|| x_{u_{N, x}^{*}}(k, x)\right|_{\mathbb{Y}} \leq \varepsilon\right\}$ satisfies the inequality $Q_{\varepsilon} \geq N-C^{\prime} / \alpha_{\ell}(\varepsilon)$.

In the case $\mathbb{Y}=\left\{x^{e}\right\}$ this property follows from Theorem 5.3 for $C^{\prime}=\max _{x \in \mathbb{X}} C+V_{N}(x)-\ell\left(x^{e}, u^{e}\right)$.

In what follows we will frequently apply Assumption 7.2 simultaneously for $p \in \mathbb{N}$ optimal trajectories with $C^{\prime}$ chosen to be the maximum of the individual constants. Denoting the respective sets by $\mathcal{P}_{\varepsilon}^{1}, \ldots, \mathcal{P}_{\varepsilon}^{p}$ we will then need that for a given $N_{0}$ the intersection $\mathcal{P}_{\varepsilon}^{\prime}:=\mathcal{P}_{\varepsilon}^{1} \cap \ldots \cap$ $\mathcal{P}_{\varepsilon}^{p} \cap\left\{0, \ldots, N-N_{0}\right\}$ contains at least one time instant $P$. Combinatorial considerations show that this is always guaranteed if each $\mathcal{P}_{\varepsilon}^{i}$ contains at least $K \geq((p-1) N+$ $\left.N_{0}+m\right) / p$ elements which in turn is guaranteed by the inequality

$$
\varepsilon \geq \alpha_{\ell}^{-1}\left(\frac{p C^{\prime}}{N-N_{0}-m}\right) .
$$

The following lemma shows an important consequence from Assumption 7.2.

Lemma 7.3 Consider a finite horizon optimal control problem satisfying Assumption 7.2 and Condition (b) from Theorem 4.2 for some $N_{0} \in \mathbb{N}$ and $\bar{\delta}>0$. Then for all $x \in \mathbb{X}, N \geq N_{0}$ and $\varepsilon \leq \bar{\delta}$ satisfying (21) with $p=1$ and $m=1$, the set $\mathcal{P}_{\varepsilon}^{\prime}=\mathcal{P}_{\varepsilon} \cap\left\{0, \ldots, N-N_{0}\right\}$ is nonempty and for each $P \in \mathcal{P}_{\varepsilon}^{\prime}$, the identity $V_{N}(x)=$ $P J_{P}\left(x, u_{N, x}^{*}\right) / N+(N-P) V_{N-P}(y) / N+R_{1}(N, \varepsilon)$ holds, where $u_{N, x}^{*} \in \mathbb{U}^{N}(x)$ is the optimal control for initial value $x$ and horizon $N, y$ is an arbitrary point in $\mathbb{Y}$ and the remainder term $R_{1}(N, \varepsilon)$ satisfies $\left|R_{1}(N, \varepsilon)\right| \leq$ $\gamma_{V}(\varepsilon) / N$.

Proof: The fact $\mathcal{P}_{\varepsilon}^{\prime} \neq \emptyset$ follows from the derivation of (21). For the proof of the claimed identity, from the optimality principle we get $V_{N}(x)=\frac{P}{N} J_{P}\left(x, u_{N, x}^{*}\right)+$ $\frac{N-P}{N} V_{N-P}\left(x_{u_{N, x}^{*}}(P, x)\right)$. Now Assumption 7.2 and the choice of $N$ and $P$ imply $\left|x_{u_{N, x}^{*}}(P, x)\right|_{\mathbb{Y}} \leq \varepsilon<\bar{\delta}$ from which by Condition (b) of Theorem 4.2 we can conclude $\left|V_{N-P}\left(x_{u_{N, x}^{*}}(P, x)\right)-V_{N-P}(y)\right| \leq \gamma_{V}(\varepsilon) /(N-P)$. Here $y \in \mathbb{Y}$ is chosen such that $\left\|x_{u_{N, x}^{*}}(P, x)-y\right\| \leq$ $\left|x_{u_{N, x}^{*}}(P, x)\right|_{\mathbb{Y}}$ holds but since Condition (b) of Theorem 4.2 implies that $V_{N-P}$ is constant on $\mathbb{Y}$ it can actually be chosen arbitrarily. Combining this inequalities with the identity for $V_{N}$, above, yields the assertion.

By Assumption 7.2, $\left.u_{N, x}^{*}\right|_{\{0, \ldots, P-1\}}$ lies in $\overline{\mathbb{U}}^{P}(x, \varepsilon):=$ $\left\{\left.u \in \mathbb{U}^{P}(x)|| x_{u}(P, x)\right|_{\mathbb{Y}} \leq \varepsilon\right\}$ for all $P \in \mathcal{P}_{\varepsilon}$. The following lemma shows a property of this set.
Lemma 7.4 Assume that the function $\lambda$ is constant on $\mathbb{Y}$ and Lipschitz with constant $L_{\lambda}$ in the ball $\mathcal{B}_{\bar{\delta}}(\mathbb{Y})$ with radius $\bar{\delta}>0$ around $\mathbb{Y}$. Then for all $u \in \overline{\mathbb{U}}^{P}(x, \varepsilon)$ with $\varepsilon<$ $\bar{\delta}$ the identity $\widetilde{J}_{P}(x, u)=J_{P}(x, u)+\lambda(x) / P-\lambda(y) / P+$ $R_{2}(u, P, \varepsilon)$ holds with $\left|R_{2}(u, P, \varepsilon)\right| \leq L_{\lambda} \varepsilon / P$.

Proof: The assertion follows from $\widetilde{J}_{P}(x, u)=J_{P}(x, u)+$ $\lambda(x) / P-\lambda\left(x_{u}(P, x)\right) / P=J_{P}(x, u)+\lambda(x) / P-$ $\lambda(y) / P+R_{2}(u, P, \varepsilon)$ with $\left|R_{2}(u, P, \varepsilon)\right|=\mid \lambda(y) / P-$ $\lambda\left(x_{u}(P, x)\right) /\left.P\left|\leq L_{\lambda}\right| x_{u}(P, x)\right|_{\mathbb{Y}} / P \leq L_{\lambda} \varepsilon / P$, where $y \in \mathbb{Y}$ is chosen as in the proof of Lemma 7.3 and the Lipschitz property can be used because $\left|x_{u}(P, x)\right|_{\mathbb{Y}} \leq \varepsilon<\bar{\delta}$ holds.

Now we can prove the lemma about the averaged functionals along the optimal trajectories. In this lemma we assume that Assumption 7.2 holds for both the original and the modified optimal control problem and denote the respective sets of time instants by $\mathcal{P}_{\varepsilon}$ and $\widetilde{\mathcal{P}}_{\varepsilon}$.

Lemma 7.5 Assume that the assumptions of Lemma 7.3 hold for some $\bar{\delta}>0$ for both the original and the modified problem, that (21) holds for $p=2$ and $m=1$ and that the assumption of Lemma 7.4 holds with the same $\bar{\delta}>0$. Then $\mathcal{P}_{\varepsilon}^{\prime}:=\mathcal{P}_{\varepsilon} \cap \widetilde{\mathcal{P}}_{\varepsilon} \cap\left\{0, \ldots, N-N_{0}\right\} \neq \emptyset$ and for each $P \in \mathcal{P}_{\varepsilon}^{\prime}$ the identity $\widetilde{J}_{P}\left(x, \tilde{u}_{N, x}^{*}\right)=J_{P}\left(x, u_{N, x}^{*}\right)+$ $\lambda(x) / P-\lambda(y) / P+R_{3}(P, \varepsilon)$ holds with remainder term bounded by $\left|R_{3}(P, \varepsilon)\right| \leq 4\left(\gamma_{V}(\varepsilon)+L_{\lambda} \varepsilon\right) / P$.

Proof: First note that the choice of $\varepsilon$ via (21) guarantees $\mathcal{P}_{\varepsilon}^{\prime} \neq \emptyset$. We pick $P \in \mathcal{P}_{\varepsilon}^{\prime}$ and observe that the assertion of Lemma 7.3 holds for this $P$ for the original as well as for the modified problem.

Now for an arbitrary $u \in \overline{\mathbb{U}}^{P}(x, \varepsilon)$ with $\varepsilon<\bar{\delta}$ we define a new control sequence $u_{1} \in \overline{\mathbb{U}}^{N}(x)$ by setting $u_{1}(k)=u(k)$ for $k=0, \ldots, P-1$ and $u_{1}(k)=u_{N-P, x_{u}(P, x)}^{*}(k-P)$ for $k=P, \ldots, N-1$. With the same arguments as in the proof of Lemma 7.3 one sees that the identity $J_{N}\left(x, u_{1}\right)=P J_{P}(x, u) / N+(N-$ P) $V_{N-P}(y) / P+R_{4}(u, N, \varepsilon)$ holds with $\left|R_{4}(u, N \varepsilon)\right| \leq$ $\gamma_{V}(\varepsilon) / N$. This implies $P J_{P}(x, u) / N \geq V_{N}(x)-(N-$ $P) V_{N-P}(y) / P-R_{4}(u, N, \varepsilon)$ with remainder terms satisfying the same bounds.

Combining this estimate with the one from Lemma 7.3 yields $P J_{P}\left(x, u_{N, x}^{*}\right) / N=\inf _{u \in \overline{\mathbb{U}}^{P}{ }_{(x, \varepsilon)}} P J_{P}(x, u) / N+$ $R_{1}(N, \varepsilon)+R_{5}(N, \varepsilon)$, with remainder term bounded by $\left|R_{5}(N, \varepsilon)\right| \leq \sup _{u \in \overline{\mathbb{U}}^{P}(x, \varepsilon)}\left|R_{4}(u, P, \varepsilon)\right|$. Applying the same arguments to the modified problem we likewise obtain $P \widetilde{J}_{P}\left(x, \tilde{u}_{N, x}^{*}\right) / N=\inf _{u \in \overline{\mathbb{U}}^{P}(x, \varepsilon)} P \widetilde{J}_{P}(x, u) / N+$ $\widetilde{R}_{1}(N, \varepsilon)+\widetilde{R}_{5}(N, \varepsilon)$ with remainder terms satisfying the same bounds. Applying Lemma 7.4 to both sides of the expression for $P J_{P}\left(x, u_{N, x}^{*}\right) / N$ we obtain $P \widetilde{J}_{P}\left(x, u_{N, x}^{*}\right) / N=\inf _{u \in \overline{\mathbb{U}}^{P}(x)} P \widetilde{J}_{P}(x, u) / N+$ $R_{1}(N, \varepsilon)+R_{5}(N, \varepsilon)+R_{6}(N, \varepsilon)$ where $\left|R_{6}(N, \varepsilon)\right| \leq$ 
$2 P \sup _{u \in \overline{\mathbb{U}}^{P}(x, \varepsilon)}\left|R_{2}(u, P, \varepsilon)\right| / N$ for $R_{2}$ from Lemma 7.4.

Combining the two equations for $P \widetilde{J}_{P}$ thus obtained yields $P \widetilde{J}_{P}\left(x, \tilde{u}_{N, x}^{*}\right) / N=P \widetilde{J}_{P}\left(x, u_{N, x}^{*}\right) / N-R_{1}(N, \varepsilon)-$ $R_{5}(N, \varepsilon)-R_{6}(N, \varepsilon)+\widetilde{R}_{1}(N, \varepsilon)+\widetilde{R}_{5}(N, \varepsilon)$. Now the assertion follows by applying Lemma 7.4 once more and $R_{3}$ is obtained by adding the individual remainder terms used in the proof multiplied by $N / P$.

With the help of these lemmas we can formulate the convergence theorem using $\widetilde{V}_{N}$.

Theorem 7.6 Consider the receding horizon scheme obtained from the optimal control problems with original cost $\ell$. Assume that the problem with original cost $\ell$ and the problem with modified cost $\tilde{\ell}$ satisfy Assumption 7.2 and Condition (b) from Theorem 4.2 for some $N_{0} \in \mathbb{N}$ and $\bar{\delta}>0$. Assume furthermore that $\lambda$ is constant on $\mathbb{Y}$ and Lipschitz with constant $L_{\lambda}$ in the ball $\mathcal{B}_{\bar{\delta}}(\mathbb{Y})$ with radius $\bar{\delta}>0$ around $\mathbb{Y}$.

(i) Assume that (18) holds for the receding horizon problem with original stage cost $\ell$ and that the optimal value functions $\widetilde{V}_{N}$ of the modified problem satisfy (19). Then there exists $\tilde{\delta} \in \mathcal{L}_{\mathbb{N}}$ such that for all sufficiently large $N$ the inequality $\left|x_{\mu_{N}}(k)\right|_{\mathbb{Y}} \leq \max \left\{\eta(N), \alpha^{-1}(\tilde{\delta}(N))\right\}$ holds for all $k \geq N$.

(ii) If, moreover, $\widetilde{V}_{N}$ satisfies (20), then the inequality $\left|x_{\mu_{N}}(k)\right|_{\mathbb{Y}} \leq \max \left\{\eta(N), \alpha^{-1}\left(\bar{\alpha}\left(|x|_{\mathbb{Y}}\right)+\tilde{\delta}(N)\right)\right\}$ holds for all sufficiently large $N$, all $k \in\{1, \ldots, N-1\}$ and all $x \in \mathbb{X}$ with $\widetilde{V}_{k}(x) \geq \ell_{0}$.

Proof: It is sufficient to show that there exists $\tilde{\delta} \in \mathcal{L}_{\mathbb{N}}$ such that (18) holds for $\widetilde{J}_{K}, \widetilde{V}_{N}$ and $\tilde{\delta}$, because then the desired estimates can be concluded just as in the proof of Theorem 7.1. We deduce this inequality from (18) for $J_{K}, V_{N_{-}}$and $\delta$ and the preceding lemmas. To this end, fix $\varepsilon \leq \bar{\delta}$. We are going to invoke Lemma 7.3 four times and Lemma 7.5 twice. Hence, we use Assumption 7.2 eight times and thus choose $N \in \mathbb{N}$ such that (21) holds for $p=8$ and $m=1$. This ensures that we can find a common $P \in \mathcal{P}_{\varepsilon}^{\prime}$ for all six trajectories under consideration which we will use in what follows.

Applying Lemma 7.3 to both the original and the modified problem at the points $x$ and $x_{\mu_{N}}(K)$ and Lemma 7.5 at the same points yields

$$
\begin{aligned}
& V_{N}(x)-V_{N}\left(x_{\mu_{N}}(K)\right)=\widetilde{V}_{N}(x)-\widetilde{V}_{N}\left(x_{\mu_{N}}(K)\right) \\
& -\frac{1}{N} \lambda(x)+\frac{1}{N} \lambda\left(x_{\mu_{N}}(K)\right)+R_{1}^{1}(N, \varepsilon)-R_{1}^{2}(N, \varepsilon) \\
& -\widetilde{R}_{1}^{1}(N, \varepsilon)+\widetilde{R}_{1}^{2}(N, \varepsilon)+\frac{P}{N} R_{3}^{2}(P, \varepsilon)-\frac{P}{N} R_{3}^{1}(P, \varepsilon)
\end{aligned}
$$

with $R_{1}^{i}(N, \varepsilon), \widetilde{R}_{1}^{i}(N, \varepsilon)$ and $R_{3}^{i}(P, \varepsilon), i=1,2$, from Lemma 7.3 and 7.5 , respectively.

Denoting the sum of the remainder terms by $R$ and using (15), (18) and then the identity above we obtain

$$
\begin{aligned}
& \widetilde{J}_{K}^{c l}\left(x, \mu_{N}\right)-\ell_{0} \\
&=J_{K}^{c l}\left(x, \mu_{N}\right)-\ell_{0}+\frac{1}{K} \lambda(x)-\frac{1}{K} \lambda\left(x_{\mu_{N}}(K)\right) \\
& \leq \frac{N}{K} V_{N}(x)-\frac{N}{K} V_{N}\left(x_{\mu_{N}}(K)\right)+\frac{\delta(N)}{\min \{N, K\}} \\
&+\frac{1}{K} \lambda(x)-\frac{1}{K} \lambda\left(x_{\mu_{N}}(K)\right) \\
&= \frac{N}{K} \widetilde{V}_{N}(x)-\frac{N}{K} \widetilde{V}_{N}\left(x_{\mu_{N}}(K)\right)+\frac{\delta(N)}{\min \{N, K\}}+\frac{N}{K} R
\end{aligned}
$$

An inspection of the remainder terms shows that the modulus of each of them can be bounded by a term of the form $\frac{1}{K} r(\varepsilon)$ where $r(\varepsilon) \rightarrow 0$ as $\varepsilon \rightarrow 0$. Choosing $\varepsilon$ depending on $N$ such that equality holds in (21) for $p=8$ and $m=1$, we obtain that $\varepsilon \rightarrow 0$ as $N \rightarrow \infty$ and the choice of $N_{1}$ guarantees that $\varepsilon(N) \leq \bar{\delta}$ holds for all $N \geq N_{1}$. Hence, the estimate holds for all $N \geq N_{1}$ and we can bound the sum of the error terms by a term of the form $\delta_{1}(N) / K$ with $\delta_{1}(N) \in \mathcal{L}_{\mathbb{N}}$. This proves (18) for $\widetilde{J}_{K}$ and $\widetilde{V}_{N}$ with $\tilde{\delta}(N)=\delta_{1}(N)+\delta(N)$ and thus finishes the proof.

Remark 7.7 The techniques from this section also allow to conclude near optimality of the closed loop trajectory during the transient phase. Here, "transient phase" refers to the time until the trajectory reaches a prescribed neighborhood of $\mathbb{Y}$. More precisely, for all $P, K \in \mathbb{N}$ with $P \leq K$ and $r>0$ we consider the set of control sequences $\widehat{\mathbb{U}}^{K}(x, r, P)$ for which $\left|x_{u}(k, x)\right|_{\mathbb{Y}} \leq r$ holds for $k=P, \ldots, K-1$. We show that there exists $P \in \mathbb{N}$ with $P \rightarrow \infty$ as $N \rightarrow \infty$ such that up to an error term vanishing as $N \rightarrow \infty$ the value $J_{K}^{c l}\left(x, \mu_{N}\right)$ is smaller than $J_{K}(x, u)$ for all $u \in \widehat{\mathbb{U}}^{K}(x, r, P)$ and all $K \geq N$.

To this end, we need the property of the optimal trajectories guaranteed by Lemma 6.3. Generalized to arbitrary sets $\mathbb{Y}$, this property demands that there exists $N_{1} \in \mathbb{N}$ and $\eta: \mathbb{N} \times \mathbb{R}_{0}^{+} \rightarrow \mathbb{R}_{0}^{+}$with $\eta(N, r) \rightarrow 0$ if $N \rightarrow \infty$ and $r \rightarrow 0$ such that the open loop optimal trajectories with horizon $N \geq N_{1}$ starting in $x_{1} \in \mathcal{B}_{\delta_{c}}\left(x_{e}\right)$ satisfy

$$
\left|x_{u_{N, x_{1}}^{*}}\left(k, x_{1}\right)\right|_{\mathbb{Y}} \leq \eta\left(N,\left|x_{1}\right|_{\mathbb{Y}}\right)
$$

for all $k=0, \ldots, P(N)$ with $P(N) \geq N / 2$. This property holds for the examples from Section 3. Moreover, assume that the open loop trajectories of the original problem satisfy Assumption 7.2 and that the closed loop solution $x_{\mu_{N}}$ converges to a neighborhood of $\mathbb{Y}$ with radius $r(N)$ with $r \in \mathcal{L}_{\mathbb{N}}$, as ensured, e.g., by Theorem 7.1 or Theorem 7.6. Throughout the derivation we will invoke Assumption 7.2 three times (via Lemma 7.3) and we will need that $\mathcal{P}_{\varepsilon}^{\prime}$ has at least $3\left(N-N_{0}\right) / 4$ elements. Hence, we assume that $N>0$ is large enough in order to ensure (21) for the $\varepsilon>0$ under consideration, $p=3$ and $m=\left\lceil 3\left(N-N_{0}\right) / 4\right\rceil$. 
Assume that there exists $\rho \in \mathcal{K}$ such that for all sufficiently small $r>0$ and all $x \in \mathcal{B}_{r}(\mathbb{Y})$ and $u \in \mathbb{U}$ with $f(x, u) \in \mathcal{B}_{r}(\mathbb{Y})$ the inequality

$$
\ell(x, u) \geq \ell_{0}-\rho(r)
$$

holds. Observe that also this condition is satisfied in our examples. Then for each $u \in \widehat{\mathbb{U}}^{K}(x, r, P)$ we get the estimate $\sum_{k=P}^{K-1} \ell\left(x_{u}(k, x), \mu_{N}\left(x_{\mu_{N}}(k, x)\right)\right) /(K-P) \geq$ $\ell_{0}-\rho(r(N))$ and thus $J_{K}(x, u) \geq P J_{P}(x, u) / K+(K-$ $P)\left(\ell_{0}-\rho(r)\right) / K$. Since each $u \in \widehat{\mathbb{U}}^{K}(x, r, P)$ satisfies $\left.u\right|_{\{0, \ldots, P-1\}} \in \overline{\mathbb{U}}^{P}(x, r)$, this implies

$$
\begin{aligned}
\inf _{u \in \widehat{\mathbb{U}}^{K}(x, r, P)} J_{K}(x, u) \geq & \frac{P}{K} \inf _{u \in \overline{\mathbb{U}}^{P}(x, r)} J_{P}(x, u) \\
& \left.+\frac{K-P}{K}\left(\ell_{0}-\rho(r)\right)\right) .
\end{aligned}
$$

On the other hand, applying Lemma 7.3 to $V_{N}(x)$ and $V_{N}\left(x_{\mu_{N}}(K)\right)$ in $(8)$, combining the two remainder terms in one term $R(N, \varepsilon)$ and setting $\delta(N)=\varepsilon(N-1)$ we obtain

$$
\begin{aligned}
& J_{K}^{c l}\left(x, \mu_{N}\right) \leq \frac{P}{K} J_{P}\left(x, u_{N, x}^{*}\right) \\
& -\frac{P}{K} J_{P}\left(x_{\mu_{N}}(K), u_{N, x_{\mu_{N}}(K)}^{*}\right)+\ell_{0}+\delta(N)+\frac{R(N, \varepsilon)}{K} .
\end{aligned}
$$

Here we choose $P \in \mathcal{P}_{\varepsilon}^{\prime}$ with $P \leq P(N)$ from (22). Such a $P$ exists because our choice of $N$ guarantees that $\mathcal{P}_{\varepsilon}^{\prime} \subseteq$ $\left\{0, \ldots, N-N_{0}\right\}$ has at least $m=\left\lceil 3\left(N-N_{0}\right) / 4\right\rceil$ elements which implies $\min \mathcal{P}_{\varepsilon}^{\prime} \leq N-N_{0}-\left\lceil 3\left(N-N_{0}\right) / 4\right\rceil \leq$ $3\left(N-N_{0}\right) / 4 \leq N / 2$. Moreover, the choice of $m$ implies that $\mathcal{P}_{\varepsilon}^{\prime} \cap\{0, \ldots,\lceil N / 2\rceil\}$ has at least $\left\lceil\left(N-N_{0}\right) / 4\right\rceil$ elements, thus $P \geq\left(N-N_{0}\right) / 4$ and in particular $P \rightarrow \infty$ as $N \rightarrow \infty$.

Now for $K \geq N$, from (22) and (23) we obtain the inequality $J_{P}\left(x_{\mu_{N}}(K), u_{N, x_{\mu_{N}}}^{*}(K)\right) \geq \ell_{0}-\rho(\eta(N, r(N)))$ for $\eta$ from $(22)$. Hence we can conclude $J_{K}^{c l}\left(x, \mu_{N}\right) \leq$ $P J_{P}\left(x, u_{N, x}^{*}\right) / K+(K-P) \ell_{0} / K+P \rho(\eta(N, r(N))) / K+$ $\delta(N)+R(N, \varepsilon) / K$. Using the identity $P J_{P}\left(x, u_{N, x}^{*}\right) / N=$ $\inf _{u \in \overline{\mathbb{U}}^{P}{ }_{(x, \varepsilon)}} P J_{P}(x, u) / N+R_{1}(N, \varepsilon)+R_{5}(N, \varepsilon)$ derived in the proof of Lemma 7.5, defining $\varepsilon$ to be minimal with (21) with $p=3$ and $m=\left\lceil 3\left(N-N_{0}\right) / 4\right\rceil$ (which implies $\varepsilon \rightarrow 0$ as $N \rightarrow \infty$ ) and combining all error terms (which tend to 0 as $N \rightarrow \infty$ since $K \geq N$ ) in one function $\Delta \in$ $\mathcal{L}_{N}$ yields $J_{K}^{\text {cl }}\left(x, \mu_{N}\right) \leq \inf _{u \in \overline{\mathbb{U}}^{P}(x, r(N))} P J_{P}(x, u) / K+$ $(K-P) \ell_{0} / K+\Delta(N)$. Comparing this estimate with (24) and enlarging $\Delta$ in order to include the term $\rho(r(N))$, for $K \geq N$ one thus obtains

$$
J_{K}^{c l}\left(x, \mu_{N}\right) \leq \inf _{u \in \widehat{\mathbb{U}}^{K}(x, r(N), P)} J_{K}(x, u)+\Delta(N) .
$$

Thus, the value $J_{K}^{c l}\left(x, \mu_{N}\right)$ is - up to an error term of order $N$ - smaller than the value of all trajectories which end up in $\mathcal{B}_{r(N)}(\mathbb{Y})$ after at most $P$ steps.

\section{Conclusions and outlook}

We have derived conditions under which a receding horizon control scheme yields approximately optimal average infinite horizon performance for the resulting closed loop trajectories. The conditions consist of a turnpike property and suitable controllability properties and have been rigorously verified for two examples. Moreover, conditions for convergence to the optimal steady state (or more general optimal solution sets $\mathbb{Y}$ ) and approximately optimal averaged finite horizon performance during the transient phase could be obtained.

Future research will include the verification of the conditions derived in this paper for larger classes of systems, an extension to discounted infinite horizon problems, the construction of a Lyapunov function for the closed loop, the investigation of optimal periodic orbits and the derivation of conditions under which the - so far only numerically verified - convergence $N \varepsilon(N-1) \rightarrow 0$ for $\varepsilon$ from (9) (as needed in Section 7) can be guaranteed.

\section{References}

[1] R. Amrit, J. B. Rawlings, and D. Angeli, Economic optimization using model predictive control with a terminal cost, Annual Rev. Control, 35 (2011), pp. 178-186.

[2] D. Angeli, R. Amrit, And J. B. Rawlings, Receding horizon cost optimization for overly constrained nonlinear plants, in Proceedings of the 48th IEEE Conference on Decision and Control - CDC 2009, Shanghai, China, 2009, pp. 7972-7977.

[3] D. Angeli and J. B. Rawlings, Receding horizon cost optimization and control for nonlinear plants, in Proceedings of the 8th IFAC Symposium on Nonlinear Control Systems - NOLCOS 2010, Bologna, Italy, 2010, pp. 1217-1223.

[4] D. P. Bertsekas, Dynamic Programming and Optimal Control. Vol. 1 and 2., Athena Scientific, Belmont, MA, 1995.

[5] D. A. Carlson, A. B. Haurie, and A. Leizarowitz, Infinite horizon optimal control — Deterministic and Stochastic Systems, Springer-Verlag, Berlin, second ed., 1991.

[6] M. Diehl, R. Amrit, and J. B. Rawlings, A Lyapunov function for economic optimizing model predictive control, IEEE Trans. Autom. Control, 56 (2011), pp. 703-707.

[7] L. GRüNE, Optimal invariance via receding horizon control, in Proceedings of the 50th IEEE Conference on Decision and Control and European Control Conference - CDC 2011, 2011, pp. 2668-2673.

[8] L. Grüne and J. Pannek, Nonlinear Model Predictive Control. Theory and Algorithms, Springer-Verlag, London, 2011.

[9] D. Q. Mayne, J. B. Rawlings, C. V. Rao, and P. O. M. SCOKAERT, Constrained model predictive control: stability and optimality, Automatica, 36 (2000), pp. 789-814.

[10] L. W. MCKenzie, Optimal economic growth, turnpike theorems and comparative dynamics, in Handbook of Mathematical Economics, Vol. III, vol. 1 of Handbooks in Econom., North-Holland, Amsterdam, 1986, pp. 1281-1355.

[11] J. A. Primbs And V. Nevistić, Feasibility and stability of constrained finite receding horizon control, Automatica, 36 (2000), pp. 965-971.

[12] J. B. Rawlings and D. Q. Mayne, Model Predictive Control: Theory and Design, Nob Hill Publishing, Madison, 2009. 